\section{Occurrence of Campylobacter spp. in poultry meat at retail and processing plants' levels in Central Italy}

\author{
Ziad Mezher, ${ }^{1}$ Stefano Saccares, ${ }^{1}$ \\ Rita Marcianò, ${ }^{2}$ Paola De Santis, ${ }^{1}$ \\ Eda Maria Flores Rodas, ${ }^{1}$ \\ Veronica De Angelis, ${ }^{1}$ Roberto Condoleo' \\ 'Institute for Experimental Veterinary \\ Medicine of Lazio and Tuscany, Rome; \\ ${ }^{2}$ Lazio Region, Veterinary Health Section, \\ Rome, Italy
}

\section{Abstract}

Human campylobacteriosis remains the most commonly reported gastrointestinal disease in Europe and Campylobacter (C.) jejuni and $C$. coli are the two species most frequently involved in such foodborne disease. Based on the sampling plan established in the region of Lazio (Central Italy) the aim of our work was to investigate the occurrence of Campylobacter spp. in poultry meat preparations collected by the local veterinary authority at retail shops and processing plants. We also observed whether various factors such as animal species or type of product affected the isolation rate. Occurrence was significantly lower than previous surveys (12/209, 5.7\%) and chicken meat was more contaminated than turkey meat.

\section{Introduction}

Campylobacter spp. are Gram negative, microaerophilic, curved or spiral rods in the family Campylobacteriaceae. The genus Campylobacter includes more than 20 species and subspecies isolated from humans and/or animals and some of these cause a variety of infections in humans and animals as well. In particular, $C$. jejuni and $C$. coli are the pathogens most commonly involved in foodborne diseases in Europe and the USA (EFSA, 2015; Spickler, 2013; Humphrey et al., 2007).

Campylobacter spp. have been found in the intestines, oral cavity, and reproductive organs of many warm blooded animals and humans; usually infected animals are asymptomatic and since these microorganisms are spread in the environment through feces, they can also be found in soil and water (Spickler, 2013; Schaffter et al., 2004). Human infection may occur by direct contact with infected animals or by consumption of contaminated unpasteurized milk, dairy products, untreated water and raw meat. Wild and domestic birds have been considered as one of the most important reservoirs of foodborne infection for humans. In fact, Campylobacter jejuni and coli are more frequently isolated from poultry than other species and the consumption of contaminated poultry meat is the leading cause of domestically acquired foodborne illnesses in humans (Humphrey et al., 2007; EFSA, 2015).

Moreover, human campylobacteriosis remains the most commonly reported gastrointestinal disease in Europe (EFSA, 2015) and in most industrialized countries in the world (Hermans et al., 2012; Wilson et al., 2008) and broiler meat is considered to be the main source of infection (EFSA, 2015). C. jejuni and occasionally $C$. coli cause enteritis in humans; the infectious dose for Campylobacter is low (few hundred cells) and the incubation period ranges from one to ten days (Humphrey et al., 2007). The symptoms may include watery or sticky diarrhea, fever, nausea, vomiting, abdominal pain, headache and muscle pain. In some cases, Campylobacter infection may trigger severe complications, including reactive arthritis and Guillain-Barré syndrome (acute, progressing paralysis) (Spickler, 2013).

According to a review, which included several worldwide surveys (Suzuki and Yamamoto, 2009), the mean prevalence of Campylobacter spp. in retail poultry meat in Europe was $53.3 \%$. However, prevalence between countries varied greatly (from 8.1 to $80.0 \%$ ). Regarding Italy, only few studies have been carried out at retail level (Nobile et al., 2013; Sammarco et al., 2010; Parisi et al., 2007; Pezzotti et al., 2003) and prevalence ranged from 20.7 to $81.3 \%$. Such studies concerned only some regions, therefore data is missing from many Italian territories.

On the other hand, to our knowledge, the prevalence of Campylobacter spp. in poultry meat collected at the processing plant level in Italy has not yet been reported in literature while only few studies have been carried out elsewhere in the European Union; two separate studies conducted in Belgium and Germany reported, respectively, a prevalence of $22.1 \%$ in broiler fillets and up to $79.0 \%$ in different turkey meat preparations (Ghafir et al., 2007; Hamedy et al., 2007).

Due to the relevance of Campylobacter as an agent of foodborne disease, the veterinary authority of the region of Lazio established, since 2011, a specific sampling/control plan regarding poultry meat at processing plants and retail. Therefore, the purpose of our study was to report the occurrence of Campylobacter spp.. in poultry meat preparations collected from retail shops and processing plants in central Italy. We also observed whether various factors such as animal species or type of product affected the isolation rate.
Correspondence: Ziad Mezher, Institute for Experimental Veterinary Medicine of Lazio and Tuscany, via Appia Nuova 1411, 00178 Rome, Italy.

$\mathrm{Tel} / \mathrm{Fax}:+39.06 .79099360$.

E-mail: ziad.mezher@izslt.it

Key words: Campylobacter; Poultry meat; Survey; Foodborne zoonosis.

Contributions: all authors contributed equally to the manuscript.

Conflict of interest: the authors declare no potential conflict of interest.

Received for publication: 25 August 2015

Revision received: 11 January 2016.

Accepted for publication: 11 January 2016.

This work is licensed under a Creative Commons Attribution-NonCommercial 4.0 International License (CC BY-NC 4.0).

(C) Copyright Z. Mezher et al., 2016

Licensee PAGEPress, Italy

Italian Journal of Food Safety 2016; 5:5495

doi:10.4081/ijfs.2016.5495

\section{Materials and Methods}

Between 2011 and 2014, a total of 209 poultry meat samples (162 chicken, 34 turkey and 13 mixed) were collected by the local Veterinary authorities at retail shops (156) and processing plants (53) spread across the Italian region of Lazio. The proportional distribution of samples among the local veterinary authorities was carried out by taking into consideration the number of existing retail shops/processing plants within the relative territories. The plan established the sampling of only fresh meat preparations (no frozen products) irrespective of the packaging status. Samples were refrigerated and delivered within 24 hours to the laboratory. All samples were prepared according to the specific rules of ISO 6887-2 (ISO, 2003) and isolation of Campylobacter spp. was performed in accordance with ISO 10272-1 (ISO, 2006).

Briefly, the detection method consisted of inoculating $25 \mathrm{~g}$ of the sample into $225 \mathrm{ml}$ of a selective pre-enrichment medium (Bolton Broth) and incubating in a microaerophilic atmosphere (Campygen; 0xoid, Basingstoke, UK) at $37^{\circ} \mathrm{C}$ for $4-6 \mathrm{~h}$ and then at $41.5^{\circ} \mathrm{C}$ for $44 \pm 4$ h. After enrichment, the Bolton Broth was plated onto two different selective media (Modified Charcoal Cefoperazone Deoxycholate Agar and Campylobacter Selective Medium, Skirrow) and incubated for $48 \mathrm{~h}$ at $41.5^{\circ} \mathrm{C}$ in a microaerophilic atmosphere. After incubation, between one to five 
typical colonies were plated onto Columbia Blood Agar (ASC; Biolife, Milan, Italy) and incubated for $48 \mathrm{~h}$ at $41.5 \pm 1^{\circ} \mathrm{C}$ in a microaerophilic atmosphere. Campylobacter cultures were then confirmed by microscopic observation to examine the morphology and motility of isolates followed by the oxidase test, the mobility test, and a double growth test at $25 \pm 1^{\circ} \mathrm{C}$ in a microaerophilic atmosphere and at $41.5 \pm 1^{\circ} \mathrm{C}$ in aerobiosis.

Confirmation of isolates and identification of the relative Campylobacter species was performed using two different PCR qualitative assays; the first, used as a screening method, is a commercial real time PCR kit method (Adiafood Detection System Campylobacter; bioMérieux, Marcy l'Etoile, France) that enables the amplification of the target DNA using specific primers and molecular beacons designed to detect common sequences of the three thermotolerant Campylobacter species: $C$. coli, $C$. jejuni, and $C$. lari. The second, which is a multiplex PCR, was performed to distinguish between $C$.jejuni and $C$. coli using two different pairs of primers $(C$.jejuni-specific hip $O$ gene sequence of 735 bp (Linton et al., 1997 ) and a $500 \mathrm{bp}$ fragment of a $C$. coli putative aspartokinase gene (Linton et al., 1997).

The $\chi^{2}$ test was performed to evaluate the presence of a significant association $(\mathrm{P}<0.05)$ between prevalence and factors such as sampling stage, type of product (minced meat preparations versus meat preparations) or packaging status.

\section{Results}

Campylobacter spp. was isolated from twelve poultry meat samples $(12 / 209,5.7 \%)$. Table 1 shows the number of positive poultry meat samples classified by type of product. Campylobacter was detected in eleven chicken meat samples $(11 / 162,6.8 \%)$ and isolates were identified as follows: 7 were $C$. coli, 3 were $C$. jejuni species and one isolate was different from coli/jejuni species. None of the turkey meat preparations resulted positive $(0 / 34)$ while two different strains of Campylobacter (jejuni and coli) were isolated from one mixed meat preparation $(1 / 13,7.7 \%)$.

In consideration of the sampling stage, the occurrence was $6.4 \%$ for samples collected at retail (10/156) and $3.8 \%$ for those collected at the processing plants (2/53). The occurrence of the pathogen was $10.7 \%(6 / 50)$ in unpackaged products and $2.7 \%(3 / 108)$ in packaged products. The information regarding the packaging status of 39 meat preparations was not provided by the local veterinary authorities (3 positive samples, $7.1 \%$ ).

Statistical analysis did not show any association between occurrence and the factors we considered even though the packaging status was nearly significant $(\mathrm{P}=0.053)$.

\section{Discussion}

Although surveys from international literature about Campylobacter in poultry meat can differ in sampling design and testing methods, a comparison with the results of the present study can still be made with caution. Surprisingly, the occurrence is significantly lower $(5.7 \%)$ if compared with the other Italian surveys (Nobile et al., 2013; Sammarco et al., 2010; Parisi et al., 2007; Pezzotti et al., 2003); in general, it was also lower than all the prevalence studies regarding poultry meats and byproducts performed in other countries (Suzuki and Yamamoto, 2009). The reason beyond this low rate might be explained, as assumed by Nobile et al. (2013), by the fact that in recent years a higher level of attention regarding slaughter hygiene has been applied in order to reduce the risk of contamination. Furthermore, the above-mentioned studies concerned samples collected at retail whereas in this study samples were also gathered at the processing plant level where the occurrence of Campylobacter is usually lower (Padungtod and Kaneene, 2005; EFSA, 2015).

All the isolates except one proved to belong to $C$. jejuni and coli species (11/12). Similar high proportion has been also observed in several surveys (EFSA, 2015; Suzuki and Yamamoto, 2009) and underlines the relevant impact of contaminated poultry meat on public health in consideration of the major zoonotic role of such species.

The ratio between the two species has been strongly in favor of $C$. coli; a similar result has been reported by Nobile et al. (2013), while the studies from Pezzotti et al. (2003) and Sammarco et al. (2010) have reported higher jejuni isolates. In general, the international literature supports such variability of the ratio between those two species in poultry meat (Suzuki and Yamamoto, 2009).

Chicken meat preparations turned out to be more contaminated than turkey meat preparations (none of the samples resulted positive) and preparations made with mixed meat (i.e. chicken and turkey meat). This is in line with a recent EU report 2013 (EFSA, 2015) where the occurrence of Campylobacter was higher in chicken meat compared to other poultry species (mainly turkey).

Higher occurrence has been detected in samples collected at retail compared to those gathered at processing plants. Similar results have been reported in other studies and suggest that such difference might be due to cross-contamination as a result of greater handling of the products at retail (Padungtod and Kaneene, 2005; Nobile et al., 2013). Cross-contamination might also explain the higher prevalence rate that has been detected between unpackaged and packaged poultry meat products.

Minced meat preparations resulted less contaminated than other meat preparations in agreement with other studies and reports (Suzuki and Yamamoto, 2009; EFSA, 2015). On the other hand, chicken thighs were the most contaminated (6/51) probably because such cuts are most at risk in view of the anatomical proximity to the final part of the digestive tract.

\section{Conclusions}

In conclusion, considering the remarkable variability of prevalence between the Italian regions, a national-scale survey on Campylobacter spp. in poultry meat based on the same sampling design and testing method should be established in order to obtain more accurate data. In any case, the detection of Campylobacter spp. in poultry meat should remain a priority for the veterinary authorities in view of the facts that almost all isolates belonged to the two species most frequently

Table 1. Distribution of poultry meat samples according to type of product.

\begin{tabular}{lccc}
\hline Matrix information & Sample type & Total units tested (n) & Total units positive (n) \\
Whole carcasses & & 9 & 0 \\
Minced meat preparations & & & \\
& Hamburgers & 27 & 1 \\
& Fresh sausages & 15 & 0 \\
& Other & 24 & 0 \\
& Total & 66 & 1 \\
\hline Meat preparations & Wings & 9 & 0 \\
& Thighs & 51 & 6 \\
& Breasts & 42 & 2 \\
& Other & 32 & 3 \\
& Total & 134 & 11 \\
Total & & 209 & 12 \\
\hline
\end{tabular}


associated with human campylobacteriosis and that a high number of reported cases still occur each year in Europe.

\section{References}

EFSA, 2015. The European Union summary report on trends and sources of zoonoses, zoonotic agents and food-borne outbreaks in 2013. EFSA J 13:3991.

Ghafir Y, China B, Dierick K, De Zutter L, Daube G, 2007. A seven-year survey of campylobacter contamination in meat at different production stages in Belgium. Int J Food Microbiol 116:111-20.

Hamedy A, Ludewig M, Fehlhaber K, Alter T, Schlichting D, 2007. Quantitative detection of Campylobacter spp. on turkey carcasses and turkey meat. Fleischwirtschaft 87:121-4.

Hermans D, Pasmans F, Messens W, Martel A, Van Immerseel F, Rasschaert G, 2012. Poultry as a host for the zoonotic pathogen campylobacter jejuni. Vector-borne Zoonot 12:89-98.

Humphrey T, 0'Brien S, Madsen M, 2007. Campylobacters as zoonotic pathogens: a food production perspective. Int $\mathrm{J}$ Food Microbiol 117:237-57.
ISO, 2003. Microbiology of food and animal feeding stuffs - preparation of test samples, initial suspension and decimal dilutions for microbiological examination. Part 2: Specific rules for the preparation of meat and meat products. ISO Norm 68872:2003. International Organization for Standardization, Geneva, Switzerland.

ISO, 2006. Microbiology of food and animal feeding stuffs - Horizontal method for detection and enumeration of campylobacter spp. Part 1: Detection method. UNI EN ISO Norm 10272-1:2006. International Organization for Standardization, Geneva, Switzerland.

Linton D, Lawson AJ, Owen RJ, Stanley J, 1997. PCR detection, identification to species level, and fingerprinting of campylobacter jejuni and campylobacter coli direct from diarrheic samples. J Clin Microbiol 35:2568-72.

Nobile CGA, Costantino R, Bianco A, Pileggi C, Pavia M, 2013. Prevalence and pattern of antibiotic resistance of campylobacter spp. in poultry meat in southern Italy. Food Control 32:715-8.

Padungtod P, Kaneene J, 2005. Campylobacter in food animals and humans in northern Thailand. J Food Protect 68:2519-26.

Parisi A, Lanzilotta SG, Addante N, Normanno G, Di Modugno G, Dambrosio A, 2007. Prevalence, molecular characterization and antimicrobial resistance of thermophilic campylobacter isolates from cattle, hens, broilers and broiler meat in south-eastern Italy. Vet Res Commun 31:113-23.

Pezzotti G, Serafin A, Luzzi I, Mioni R, Milan M, Perin R, 2003. Occurrence and resistance to antibiotics of campylobacter jejuni and campylobacter coli in animals and meat in northeastern Italy. Int J Food Microbiol 82:281-7.

Sammarco ML, Ripabelli G, Fanelli I, Grasso GM, Tamburro M, 2010. Prevalence and biomolecular characterization of campylobacter spp. isolated from retail meat. $\mathrm{J}$ Food Protect 73:720-8.

Schaffter N, Zumstein J, Parriaux A, 2004. Factors influencing the bacteriological water quality in mountainous surface and groundwaters. Acta Hydroch Hydrob 32:225-34.

Spickler AR, 2013. Zoonotic campylobacteriosis. Available from: www.cfsph.iastate.edu/ Factsheets/pdfs/campylobacteriosis.pdf

Suzuki H, Yamamoto S, 2009. Campylobacter contamination in retail poultry meats and by-products in the world: a literature survey. J Vet Med Sci 71:255-61.

Wilson DJ, Gabriel E, Leatherbarrow AJH, Cheesbrough J, Gee S, Bolton E, 2008. Tracing the source of campylobacteriosis. PloS Genet 4:e1000203. 